\title{
High-quality grand unified theories with three generations
}

\author{
Ning Chen ${ }^{1, *}$ \\ ${ }^{1}$ School of Physics, Nankai University, Tianjin, 300071, China
}

\begin{abstract}
We extend the unitary groups beyond the $\mathrm{SU}(5)$ and $\mathrm{SU}(6)$ to look for possible grand unified theories that give rise to three-generational Standard Model fermions without the simple repetition. By demanding asymptotic free theories at short distances, we find gauge groups of $\mathrm{SU}(7), \mathrm{SU}(8)$ and $\mathrm{SU}(9)$ together with their anomaly-free irreducible representations are such candidates. Two additional gauge groups of SU(10) and SU(11) can also achieve the generational structure without asymptotic freedom. We also find these models can solve the Peccei-Quinn (PQ) quality problem which is intrinsic in the axion models, with the leading PQ-breaking operators determined from the symmetry requirement.
\end{abstract}

* chenning_symmetry@nankai.edu.cn 


\section{INTRODUCTION}

Grand Unified Theories (GUTs) [1, 2] were proposed to unify all fundamental interactions described by the Standard Model (SM). Aside from the aesthetic aspect of achieving the gauge coupling unification in its supersymmetric (SUSY) extension [3], it is pragmatic to conjecture the zeroth law of GUT, namely, a successful GUT could address all intrinsic SM puzzles and as many physical issues beyond the SM as possible, with all necessary but the minimal set of fields determined by symmetry. One such longstanding puzzle that has not been well answered is the existence of three generational SM fermions, as well as their mass hierarchies in the framework of GUTs 1 . In a seminal paper [15], Georgi suggested to extend the minimal SU(5) into larger simple Lie group $\mathrm{SU}(N)$ (with $N \geq 7$ ), and built his three laws of GUTs. Instead of simple repetition of a set of anomaly-free irreducible representations (irreps) for three times, it is argued that the three-generational structure arises from different anti-symmetric irreps of the $\mathrm{SU}(N)$.

In this paper, we investigate the possible non-minimal GUTs beyond the SU(5) and $\mathrm{SU}(6)$ that can give rise to three-generational SM fermions. The number of generations can be easily obtained according to the counting method in terms of the $\mathrm{SU}(5)$ irreps as given in Ref. [15]. It turns out that the SM fermion generations $n_{g}$ can already become three or beyond for the SU(7) group already [16]. Historically, the number of the SM fermion generations $n_{g}$ was also considered to be beyond three [17]. Meanwhile, the direct searches for the fourth-generational quarks at the Large Hadron Collider (LHC) have already excluded this possibility [18 20]. Therefore, only the non-minimal GUTs with their anomaly-free irreps that lead to $n_{g}=3$ cases will be considered in our study. In Georgi's third law, he decided that no individual irrep of the GUT group should appear more than once. Accordingly, he found that the minimal GUT group that give rise to $n_{g}=3$ is $\mathrm{SU}(11)$, with a total number of 1,023 left-handed fermions [15]. Obviously, the third law prevent the three-generational structure through the simple repetition of the anomaly-free irreps. However, this may be a too strong constraint and was not usually adopted in the later studies. In our discussions, we modify Georgi's third law in a different version proposed by Christensen and Shrock [21] in the study of the dynamical origin of the SM fermion masses. A different point of view can be made such that the global symmetries can usually emerge once the original third law was abandoned, such as in the SU(9) GUT [22]. This can be advantageous at least in two aspects. The first advantage is the emergent global symmetry, with its breaking, can be a mechanism to explain the lightness of the Higgs boson, as was discussed by Dvali [23] in the context

\footnotetext{
${ }^{1}$ Some of the previous efforts in addressing the SM fermion masses in GUTs include Refs. 4 14.
} 
of the SUSY SU(6). The other advantage is that the global U(1) symmetry can be identified as the Peccei-Quinn (PQ) symmetry [24] for the strong CP problem. The emergent $\mathrm{PQ}$ symmetry, together with both the gauge and the global symmetries, can usually constrain the mass dimensions of the PQ-breaking operators and lead to a highquality axion [22, 25, 29]. Two recent examples include the axion from the $\mathrm{SO}(10)$ [30] and the SUSY SU(6) GUT [31].

The rest of the paper is organized as follows. In Sec. II] we review Georgi's guidelines of building the non-minimal GUTs that can lead to three generations of SM fermions without simple repetition. Some other relevant results of the gauge anomaly cancellation, the Higgs representations, and the PQ quality are also setup there. Sec. III is the core of this work. We analyze all possible $\mathrm{SU}(N)$ GUTs (up to $\mathrm{SU}(11)$ ) and their anomaly-free fermion contents that can lead to three generational SM fermions according to Georgi's counting. The PQ charge assignments to Higgs fields and the corresponding PQ-breaking operators will be presented. We summarize our results and make discussions in Sec. IV]

\section{SOME GENERAL REMARKS}

\section{A. Lie group representations and Georgi's guidelines}

To facilitate the discussion, we express the fermion representations under the $\mathrm{SU}(N)$ GUT group in terms of the set of rank- $k$ anti-symmetric irrep of $[N, k]$ as follows

$$
\left\{f_{L}\right\}_{\mathrm{SU}(N)}=\sum_{k=0}^{N-1} n_{k}[N, k],
$$

with $n_{k}$ being the multiplicity. Obviously, $k=0$ corresponds to the singlet representation, and $k=1$ corresponds to the fundamental representation, and etc. The singlet representations contribute neither to the gauge anomaly, nor to the renormalization group equations (RGEs). Through out the discussion, we always denote the conjugate representation such that $\overline{[N, k]}=[N, N-k]$. It will be also useful to use a compact vector notation of

$$
\vec{n} \equiv\left(n_{0}, \ldots, n_{N-1}\right) .
$$

For a given rank- $k$ anti-symmetric irrep of $[N, k]$, its dimension and trace invariants are

$$
\begin{aligned}
\operatorname{dim}([N, k]) & =\frac{N !}{k !(N-k) !}, \\
T([N, k]) & =\frac{(N-2) !}{2(k-1) !(N-k-1) !} .
\end{aligned}
$$


From Cartan's classification, it is well-known that the only possible Lie groups for non-minimal GUTs beyond the $\mathrm{SU}(5)$ or $\mathrm{SO}(10)$ are

$$
\mathrm{SU}(N) \quad(N \geq 6), \quad \mathrm{SO}(4 k+2) \quad(k \geq 3), \quad E_{6} .
$$

Since the exceptional group of $E_{6}$ has fixed rank, it is impossible to consider further extensions. For any irrep under these Lie groups, one can always decompose it under the subgroup of the $\mathrm{SU}(5)$. For example, the fundamental representation of the $\mathrm{SU}(N)$ can be decomposed as

$$
[N, 1]=(N-5) \times[5,0] \oplus[5,1] .
$$

The decompositions of the higher irreps can be obtained by LieART [32]. For an SU(N) GUT, its fermion contents can be generally decomposed in terms of the $\mathrm{SU}(5)$ irreps as follows

$$
\left\{f_{L}\right\}_{\mathrm{SU}(N)}=n_{0}[5,0]+n_{1}[5,1]+n_{2}[5,2]+n_{3}[5,3]+n_{4}[5,4] .
$$

The anomaly cancellation condition leads to the following relation to the multiplicities

$$
n_{1}+n_{2}=n_{3}+n_{4}
$$

In Ref. [15], Georgi argued that the counting of the SM fermion generations is equivalent to the counting of the multiplicity of the residual $\mathrm{SU}(5)$ irreps of $[5,2] \oplus[5,4]$, which is

$$
n_{g}=n_{2}-n_{3}=n_{4}-n_{1} .
$$

Note that the counting of the SM fermion generations in Eq. (9) does not rely on the realistic gauge symmetry breaking patterns. Based on Georgi's counting, it turned out that any GUT with orthogonal groups larger than the $\mathrm{SO}(10)$ essentially leads to $n_{g}=0$. This can be understood by decomposing the 16-dimensional $\mathrm{SO}(10)$ Weyl fermions under the $\mathrm{SU}(5)$ as

$$
16_{\mathbf{F}}=\mathbf{1}_{\mathbf{F}} \oplus \overline{\mathbf{5}_{\mathbf{F}}} \oplus \mathbf{1 0}_{\mathbf{F}}
$$

The Weyl fermions from larger orthogonal groups are always decomposed under the $\mathrm{SO}(10)$ in pairs of $\mathbf{1 6}_{\mathbf{F}} \oplus \overline{\mathbf{1 6}_{\mathbf{F}}}$, and this can only lead to $n_{g}=0$.

Georgi's third law requires that not any representation of $[N, k]$ should appear more than once, which means $n_{k}=0$ or $n_{k}=1$ in Eq. (1). This leads to a consequence that no global symmetry can emerge from the corresponding fermion setup. Instead, we adopt an alternative criterion by Christensen and Shrock [21], namely, the greatest common 
divisor of $\left\{n_{k}\right\}$ is not greater than unity. Therefore, one can expect the global symmetry of

$$
\mathcal{G}_{\text {global }}=\prod_{\left\{k^{\prime}\right\}}\left[\mathrm{SU}\left(n_{k^{\prime}}\right) \otimes \mathrm{U}(1)_{k^{\prime}}\right]
$$

for all irreps of $\left[N, k^{\prime}\right]$ that appear more than once. This can be viewed as a generalization of the global symmetry in the rank-2 anti-symmetric theory of $\mathrm{SU}(N+4)$ by Dimopoulos, Raby, and Susskind (DRS) [33] . The U(1) components of the global symmetry (11) can be identified as the global PQ symmetry, which are likely to lead to high-quality axion [31. In this regard, the modified criterion of the fermion assignments is likely to solve the long-standing PQ quality problem [22, 25] 29] in the framework of GUT.

\section{B. Gauge anomaly cancellation}

To have an anomaly-free non-minimal GUT, we have to solve the following Diophantine equation

$$
\vec{n} \cdot \vec{A}=0,
$$

with the $N$-dimensional anomaly vector [34, 35] being

$$
\begin{aligned}
& \vec{A}=(A([N, 0]), A([N, 1]), \ldots, A([N, N-1])), \\
& A([N, 0])=0, \quad A([N, k])=\frac{(N-2 k)(N-3) !}{(N-k-1) !(k-1) !}(k>0) .
\end{aligned}
$$

The property that the anomaly of a given irrep and its conjugate cancel each other is apparent in Eq. (14). Also, the self-conjugate representations must be anomaly-free such that $A\left(\left[N, \frac{N}{2}\right]\right)=0$ for $N$ being even. Thus, the anomaly vector can be expressed as

$$
\vec{A}=\left(0, A([N, 1]), \ldots, A\left(\left[N,\left[\frac{N}{2}\right]\right]\right),-A\left(\left[N,\left[\frac{N}{2}\right]\right]\right), \ldots,-A([N, 1])\right) .
$$

In practice, one has to decompose the $\mathrm{SU}(N)$ fermion representations from $[N, 1]$ to $\left[N,\left[\frac{N}{2}\right]\right]$ under the $\mathrm{SU}(5)$ in order to count the generations.

\section{The Higgs representations}

Once the fermion contents are determined for a particular non-minimal GUT, the Higgs fields can be determined by the following criteria 
1. The GUT symmetry breaking of $\mathrm{SU}(N) \rightarrow \mathrm{SU}(m) \otimes \mathrm{SU}(N-m) \otimes \mathrm{U}(1)$ with $m=\left[\frac{N}{2}\right]$ is always assumed at its first stage, which requires an adjoint Higgs field [36]. The other possible symmetry breaking of $\mathrm{SU}(N) \rightarrow \mathrm{SU}(N-1)$ (with $N \geq 6$ ) at the first stage is very likely to lower the proton lifetime predictions, and thus bring tension with the current experimental constraint to the proton lifetime from the Super-Kamionkande [37].

2. All possible gauge-invariant Yukawa couplings, which also respect the global symmetry in Eq. (11), can be formed.

3. Higgs fields to achieve any intermediate symmetry breaking stages are necessary, where their proper irreps contain the SM-singlet directions.

4. Only the Higgs fields with the minimal dimensions are taken into account.

Before proceeding to the more realistic models, we display the Higgs fields in the SU(6) GUT as an example. Its minimal anomaly-free fermion contents and decomposition under the $\mathrm{SU}(5)$ are

$$
\begin{aligned}
\left\{f_{L}\right\}_{\mathrm{SU}(6)} & =[6,2] \oplus 2 \times[6,5]=\mathbf{1 5}_{\mathbf{F}} \oplus 2 \times \overline{\mathbf{6}_{\mathbf{F}}} \\
& =2 \times[5,0] \oplus[5,1] \oplus[5,2] \oplus 2 \times[5,4] .
\end{aligned}
$$

The $[5,1]$, one of the $[5,4]$, as well as two singlets of $[5,0]$ obtain their masses at an intermediate symmetry-breaking scale. The remaining fermions contain precisely one generational SM fermions of $[5,2] \oplus[5,4] \approx[5,2] \oplus \overline{[5,1]}$. Apparently, the minimal $\mathrm{SU}(6)$ GUT has a global symmetry of $\mathrm{SU}(2)_{\overline{\mathbf{6}_{\mathbf{F}}}} \otimes \mathrm{U}(1)_{\mathrm{PQ}}$ and is a one-generational model according to Georgi's counting. The gauge-invariant and $\mathrm{SU}(2) \overline{\mathbf{6}_{\mathbf{F}}}$-invariant Yukawa coupling can be expressed as follows

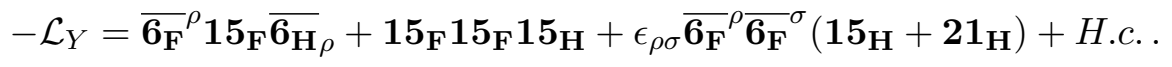

with the minimal set of Higgs fields. Of course, a 35-dimensional adjoint Higgs field is necessary to achieve the first-stage GUT symmetry breaking of SU(6) $\rightarrow$ $\mathrm{SU}(3)_{c} \otimes \mathrm{SU}(3)_{W} \otimes \mathrm{U}(1)_{X}[31,38,39$. It turns out that the VEVs from Higgs fields of $\left(\mathbf{1}, \overline{\mathbf{3}},-\frac{1}{3}\right)_{\mathbf{H}, \rho} \subset \overline{\mathbf{6}}_{\mathbf{H}}$ and $\left(\mathbf{1}, \mathbf{6},+\frac{2}{3}\right)_{\mathbf{H}} \subset \mathbf{2 1}_{\mathbf{H}}$ can be responsible for the intermediate symmetry breaking of $\mathrm{SU}(3)_{c} \otimes \mathrm{SU}(3)_{W} \otimes \mathrm{U}(1)_{X} \rightarrow \mathrm{SU}(3)_{c} \otimes \mathrm{SU}(2)_{W} \otimes \mathrm{U}(1)_{Y}$ [31].

\section{The asymptotic freedom (AF)}

The GUTs with their earliest versions are usually asymptotically free above the unification scale. However, there was no definite answer whether the AF should be retained. 
An alternative criterion is to have an asymptotic safe theory, which reaches a fixed point at the short distance [40, 41]. In general, the analysis of the asymptotic safe theories involves the RGEs of gauge couplings, as well as Yukawa and Higgs self couplings. This can only be performed for individual theory by specifying the symmetry breaking patterns. In the non-minimal GUTs, the AF is likely to be violated since the trace invariants of the rank-2 and rank-3 anti-symmetric representations scale as $T([N, 2]) \sim N$ and $T([N, 3]) \sim N^{2}$. Previously, this was also considered in the SU(11) model [42], but with only fermions taken into account. In our discussions below, we study the short-distance behavior for non-minimal GUTs up to SU(11), with their minimal fermion setup. It turns out the minimal models in $\mathrm{SU}(10)$ and $\mathrm{SU}(11)$ violate the $\mathrm{AF}$, and thus careful analysis of their unification couplings and scales are necessary for these two cases. The one-loop $\beta$ coefficients are obtained by including both fermions and Higgs fields as follows

$$
b_{1}=-\frac{11}{3} C_{2}(\mathcal{G})+\frac{4}{3} \kappa \sum_{f} T\left(\mathcal{R}_{f}\right)+\frac{1}{3} \eta \sum_{s} T\left(\mathcal{R}_{s}\right),
$$

with $\kappa=1(1 / 2)$ for Dirac (Weyl) fermions, and $\eta=1(1 / 2)$ for complex (real) scalars. For the adjoint Higgs fields, we always consider them to be real for the non-SUSY case. The AF can be determined by whether $b_{1}<0$ or not.

\section{E. The PQ quality and axion}

The global PQ symmetry has an intrinsic problem known as the PQ quality 22, 2529]. In general, global symmetries are not fundamental but arise with the underlying gauge theories. They are believed to be broken by quantum gravity effects in the form of the following dimension- $2 m+n$ operator

$$
\mathcal{O}_{\mathrm{RQ}}^{d=2 m+n}=k \frac{|\Phi|^{2 m} \Phi^{n}}{M_{\mathrm{pl}}^{2 m+n-4}} .
$$

The size of PQ-breaking is constrained such that the minima of the QCD effective potential induced by axion should satisfy $\left|\left\langle a / f_{a}\right\rangle\right| \lesssim 10^{-10}$, which leads to a PQ quality constraint of

$$
\frac{f_{a}^{d}}{M_{\mathrm{pl}}^{d-4}} \lesssim 10^{-10} \Lambda_{\mathrm{QCD}}^{4} .
$$

It turns out that the mass dimension in Eq. (19) should be $d \gtrsim 9$ in order to have a reasonable axion decay constant $f_{a} \sim \mathcal{O}\left(10^{12}\right) \mathrm{GeV}$ without much fine tuning of the coefficient $k$ [26 28] in Eq. (19). Without knowing underlying symmetry origin of the $\Phi$ field, there is generally no reason to forbid any PQ-breaking operators with $d \lesssim 9$. 
Previous studies of the axion in the GUT [43 46] were made in both SU(5) and $\mathrm{SO}(10)$, where the global PQ symmetry was introduced by hand. Therefore, the issue of $\mathrm{PQ}$ quality was still present. Recent discussions of the PQ quality problem in the frame of GUT include the $\mathrm{SO}(10)$ [30] and $\mathrm{SU}(6)$ [31] cases. In the $\mathrm{SO}(10)$ GUT, the author made use of the generational symmetry in the limit of vanishing Yukawa couplings. A dimension-9 gauge-invariant operator to produce a high-quality axion was found, which is made up of Higgs fields for the intermediate symmetry breaking. In the minimal SU(6) GUT, it already possesses a global DRS symmetry as in Eq. (11). With the SUSY extensions, the authors [31] found a dimension-6 operator that lead to a highquality axion. Therefore, it becomes suggestive that the GUTs beyond the minimal versions are likely to solve the PQ-quality problem, with their local and emergent global symmetries. In the context of GUTs, the PQ-breaking operators can be formed by Higgs fields that develop vacuum expectation values (VEVs) at both the electroweak (EW) scale of $v_{\mathrm{EW}}$ and the $\mathrm{PQ}$ symmetry-breaking scale of $f_{a}$. This further alleviates the $\mathrm{PQ}$ quality constraint in Eq. 200 even when $d<9$. For example in the minimal SUSY SU(6) GUT [31], such a PQ-breaking operator turns out to be $\mathcal{O}_{\mathrm{RQ}}^{d=6}=\left(\epsilon_{\alpha \beta}{\overline{\mathbf{6}_{\mathbf{H}}}}^{\alpha}{\overline{\mathbf{6}_{\mathbf{H}}}}^{\beta} \mathbf{1 5}_{\mathbf{H}}\right)^{2}$, with $\alpha=1,2$. The PQ quality constraint can be fulfilled when setting $\left\langle\overline{\mathbf{6}} \mathbf{H}^{1}\right\rangle \sim\left\langle\mathbf{1 5}_{\mathbf{H}}\right\rangle \simeq v_{\mathrm{EW}}$ and $\left\langle{\overline{\mathbf{6}_{\mathbf{H}}}}^{2}\right\rangle \simeq f_{a}$. A natural question one can raise is whether the PQ quality constraint can be generally satisfied in the non-minimal GUTs with $n_{g}=3$. Impressively, we find this generally holds with proper assignment of the PQ charges to the Higgs fields.

The probes of the axion rely on the axion-photon effective coupling of

$$
C_{a \gamma \gamma}=\frac{E}{N_{\mathrm{SU}(3)_{c}}}-1.92 .
$$

For the GUTs, there is a universal prediction to the factor $E / N_{\mathrm{SU}(3)_{c}}=8 / 3$. The color anomaly factor of $N_{\mathrm{SU}(3)_{c}}$ relates the axion decay constant with the associate symmetrybreaking scale as $v_{\mathrm{SB}}=\left|2 N_{\mathrm{SU}(3)_{c}}\right| f_{a}$, and also determines the domain wall number as $N_{\mathrm{DW}}=2 N_{\mathrm{SU}(3)_{c}}$. In practice, one does not need to derive the factor by analyzing the symmetry breaking patterns. Instead, this can be obtained by using the 't Hooft anomaly matching condition [47] of

$$
N_{\mathrm{SU}(3)_{c}}=N_{\mathrm{SU}(N)}=\sum_{\mathbf{F}} T\left(\mathcal{R}_{\mathbf{F}}\right) \mathrm{PQ}\left(\mathcal{R}_{\mathbf{F}}\right) .
$$

Notice that in our current study, the physical axion does not arise at the GUT scale of $\sim 10^{16} \mathrm{GeV}$. Instead, it arises from the phases of Higgs fields that are responsible for the intermediate symmetry breaking scale, with necessary orthogonality conditions imposed. One such example can be found in the minimal SUSY SU(6) GUT [31. We focus on the PQ-breaking operators in the non-minimal GUTs, while the constructions of the physical axion in the specific GUT model will be left for future work. 


\section{THE RESULTS}

In this section, we obtain our results of the $\mathrm{SU}(N)$ GUTs that lead to $n_{g}=3$. Examples include $\mathrm{SU}(7), \mathrm{SU}(8)$, and $\mathrm{SU}(9)$ groups, where the $\mathrm{AF}$ can be achieved. We also find that the higher groups of $\mathrm{SU}(10)$ and $\mathrm{SU}(11)$ with their minimal irreps cannot achieve the AF condition. For each case, we also look for the possible gauge-invariant and PQ-breaking operators. With proper PQ charge assignment at the GUT scale, we show that the PQ quality problem can be generally avoided in each model.

\section{A. The $\mathrm{SU}(7)$}

For the SU(7) group, the anomaly vector in Eq. (13) reads

$$
\vec{A}=(0,1,3,2,-2,-3,-1) .
$$

The decompositions of the SU(7) irreps under the SU(5) are the following

$$
\begin{aligned}
\mathbf{7} & =[7,1]=2 \times[5,0] \oplus[5,1], \\
\mathbf{2 1} & =[7,2]=[5,0] \oplus 2 \times[5,1] \oplus[5,2], \\
\mathbf{3 5} & =[7,3]=[5,1] \oplus 2 \times[5,2] \oplus[5,3] .
\end{aligned}
$$

There are two possibilities for $n_{g}=3$, namely,

$$
\begin{aligned}
\left\{f_{L}\right\}_{\mathrm{SU}(7)}^{\mathrm{A}} & =2 \times[7,2] \oplus[7,3] \oplus 8 \times[7,6] \\
& =2 \times \mathbf{2 1}_{\mathbf{F}} \oplus \mathbf{3 5}_{\mathbf{F}} \oplus 8 \times \overline{\mathbf{7}_{\mathbf{F}}}, \quad \operatorname{dim}_{\mathbf{F}}=133, \\
\mathcal{G}_{\text {global }}^{\mathrm{A}} & =\left[\mathrm{SU}(8)_{\overline{\mathbf{F}_{\mathbf{F}}}} \otimes \mathrm{U}(1)_{\mathrm{PQ}}\right] \otimes\left[\mathrm{SU}(2) \otimes \mathrm{U}(1)^{\prime}\right], \\
\left\{f_{L}\right\}_{\mathrm{SU}(7)}^{\mathrm{B}} & =[7,2] \oplus 2 \times[7,3] \oplus 7 \times[7,6] \\
& =\mathbf{2 1}_{\mathbf{F}} \oplus 2 \times \mathbf{3 5}_{\mathbf{F}} \oplus 7 \times \overline{\mathbf{7}_{\mathbf{F}}}, \quad \operatorname{dim}_{\mathbf{F}}=140, \\
\mathcal{G}_{\text {global }}^{\mathrm{B}} & =\left[\mathrm{SU}(7)_{\overline{\mathbf{7}_{\mathbf{F}}}} \otimes \mathrm{U}(1)_{\mathrm{PQ}}\right] \otimes\left[\mathrm{SU}(2) \otimes \mathrm{U}(1)^{\prime}\right] .
\end{aligned}
$$

Since the number of fermions in two cases only differ by less than 10, we determine to consider both possibilities. Note in passing, a recent study [48] suggests that the $\mathrm{SU}(7)$ model can be suppress the proton decay with the proper embedding of the SM fermions.

The Higgs sector of two $\mathrm{SU}(7)$ models is determined by the fermions and the global symmetries in Eq. (25) as follows

$$
\begin{aligned}
& \{H\}_{\mathrm{SU}(7)}^{\mathrm{A}}=8 \times \overline{\mathbf{2 1}_{\mathbf{H}}} \oplus \mathbf{7}_{\mathbf{H}} \oplus 2 \times \mathbf{2 1}_{\mathbf{H}} \oplus \mathbf{3} \mathbf{5}_{\mathbf{H}}\left[\oplus \mathbf{4 8}_{\mathbf{H}}\right], \\
& \{H\}_{\mathrm{SU}(7)}^{\mathrm{B}}=7 \times \overline{\mathbf{7}_{\mathbf{H}}} \oplus \mathbf{7}_{\mathbf{H}} \oplus 2 \times \mathbf{2 1}_{\mathbf{H}} \oplus \mathbf{3 5}_{\mathbf{H}}\left[\oplus \mathbf{4} \mathbf{8}_{\mathbf{H}}\right] .
\end{aligned}
$$


Here and below, we use the square brackets to denote the real adjoint Higgs fields for the GUT scale symmetry breaking. By using the fermions and Higgs fields in Eqs. 25) and (26), we find that $b_{1}^{A}=-5$ and $b_{1}^{B}=-\frac{55}{6}$. Thus both the $\mathrm{SU}(7)-\mathrm{A}$ and the $\mathrm{SU}(7)-\mathrm{B}$ model are asymptotically free. The gauge-invariant Yukawa couplings are

$$
\begin{aligned}
-\mathcal{L}_{Y}^{\mathrm{A}} & =\sum_{\rho=1}^{8} \overline{\mathbf{7}_{\mathbf{F}}^{\rho}} \mathbf{3 5}_{\mathbf{F}} \overline{\mathbf{2 1}} \mathbf{H}_{\mathbf{H} \rho}+\sum_{\dot{\rho}=1,2} \mathbf{2} \mathbf{1}_{\mathbf{F}}^{\dot{\rho}} \mathbf{3 5}_{\mathbf{F}} \mathbf{2} \mathbf{1}_{\mathbf{H} \dot{\rho}} \\
& +\epsilon_{\dot{\rho} \dot{\sigma}} \mathbf{2 1}_{\mathbf{F}}{ }^{\dot{\rho}} \mathbf{2 1}_{\mathbf{F}}{ }^{\dot{\sigma}} \mathbf{3} \mathbf{5}_{\mathbf{H}}+\mathbf{3 5}_{\mathbf{F}} \mathbf{3} \mathbf{5}_{\mathbf{F}} \mathbf{7}_{\mathbf{H}}+H . c . \\
-\mathcal{L}_{Y}^{\mathrm{B}} & =\sum_{\rho=1}^{7} \overline{\mathbf{7}_{\mathbf{F}}^{\rho}} \mathbf{2 1}_{\mathbf{F}} \overline{\mathbf{7}_{\mathbf{H} \rho}}+\sum_{\dot{\rho}=1,2} \mathbf{3} \mathbf{5}_{\mathbf{F}}^{\dot{\rho}} \mathbf{2} \mathbf{1}_{\mathbf{F}} \mathbf{2} \mathbf{1}_{\mathbf{H} \dot{\rho}} \\
& +\epsilon_{\dot{\rho} \dot{\sigma}} \mathbf{3 5 _ { \mathbf { F } }}{ }^{\dot{\rho}} \mathbf{3 5}_{\mathbf{F}}{ }^{\dot{\sigma}} \mathbf{7}_{\mathbf{H}}+\mathbf{2 1}_{\mathbf{F}} \mathbf{2 1}_{\mathbf{F}} \mathbf{3} \mathbf{5}_{\mathbf{H}}+H . c .
\end{aligned}
$$

\begin{tabular}{c|ccc|ccccc}
\hline \hline $\mathrm{SU}(7)-\mathrm{A}$ & $\overline{\mathbf{7}_{\mathbf{F}}}$ & $\mathbf{2 1}_{\mathbf{F}}$ & $\mathbf{3 5} \mathbf{5}_{\mathbf{F}}$ & $\overline{\mathbf{2 1}_{\mathbf{H}}}$ & $\mathbf{7}_{\mathbf{H}}$ & $\mathbf{2 1}_{\mathbf{H}}$ & $\overline{\mathbf{5 5}_{\mathbf{H}}}$ & $\mathbf{3 5}_{\mathbf{H}}$ \\
\hline $\mathrm{PQ}$ charges & 1 & 0 & 0 & -1 & 0 & 0 & 0 & 0 \\
$\mathrm{SU}(8)_{\overline{\mathbf{7}}}$ & $\square$ & 1 & 1 & $\bar{\square}$ & 1 & 1 & 1 & 1 \\
$\mathrm{SU}(2)$ & 1 & $\square$ & 1 & 1 & 1 & $\square$ & $\square$ & 1 \\
\hline \hline $\mathrm{SU}(7)-\mathrm{B}$ & $\overline{\mathbf{7}_{\mathbf{F}}}$ & $\mathbf{3 5}_{\mathbf{F}}$ & $\mathbf{2 1}_{\mathbf{F}}$ & $\overline{\mathbf{7}_{\mathbf{H}}}$ & $\mathbf{3 5}_{\mathbf{H}}$ & $\overline{\mathbf{2 1}_{\mathbf{H}}}$ & $\mathbf{2 1}_{\mathbf{H}}$ & $\mathbf{7}_{\mathbf{H}}$ \\
\hline $\mathrm{PQ}$ charges & 1 & 0 & 0 & -1 & 0 & 0 & 0 & 0 \\
$\mathrm{SU}(7)_{\overline{\mathbf{7}}}$ & $\square$ & 1 & 1 & $\overline{0}$ & 1 & 1 & 1 & 1 \\
$\mathrm{SU}(2)$ & 1 & $\square$ & 1 & 1 & 1 & $\bar{\square}$ & $\bar{\square}$ & 1 \\
\hline \hline
\end{tabular}

Table I. The PQ charge assignments and the representations of the fermions and Higgs fields under their global symmetries for two $\mathrm{SU}(7)$ unification models.

We assign the PQ charges for all SU(7) fermions and Higgs fields in Tab. If The PQ charges cannot be uniquely determined from the PQ neutrality of the Yukawa couplings (27). Therefore, we assign the PQ charges by removing the possible dangerous PQ-breaking operators with low mass dimensions. In the $\mathrm{SU}(7)-\mathrm{A}$, one may assign $\mathrm{PQ}\left(\mathbf{2 1}_{\mathbf{F}}\right)=q_{1}$ and $\mathrm{PQ}\left(\mathbf{3 5}_{\mathbf{F}}\right)=q_{2}$. Accordingly, it is easy to find two following PQbreaking operators

$$
\begin{aligned}
& \mathcal{O}_{\mathrm{RQ}}^{d=5}=\epsilon^{\dot{\rho} \dot{\sigma}} \mathbf{2 1}_{\mathbf{H} \dot{\rho}} \mathbf{2 1}_{\mathbf{H} \dot{\sigma}} \mathbf{7}_{\mathbf{H}}{ }^{3}, \quad \Delta \mathrm{PQ}=-2 q_{1}-8 q_{2} \\
& \mathcal{O}_{\mathrm{RQ}}^{d=3}=\epsilon^{\dot{\rho} \dot{\sigma}} \mathbf{2 1}_{\mathbf{H} \dot{\rho}} \overline{\mathbf{3 5}_{\mathbf{H} \dot{\sigma}}} \mathbf{7}_{\mathbf{H}}, \quad \Delta \mathrm{PQ}=-2 q_{1}-4 q_{2} .
\end{aligned}
$$

These two operators would better be PQ-neutral due to their mass dimensions, and this leads to $q_{1}=q_{2}=0$. Similarly in the $\mathrm{SU}(7)$-B and larger groups below, we find the corresponding PQ charge assignments. 
The color and electromagnetic anomaly factors, and domain wall numbers according to Eq. (22) are given by

$$
\begin{aligned}
& \mathrm{SU}(7)-\mathrm{A}: N_{\mathrm{SU}(3)_{c}}=4, \quad E=\frac{32}{3}, \quad N_{\mathrm{DW}}=8, \\
& \mathrm{SU}(7)-\mathrm{B}: N_{\mathrm{SU}(3)_{c}}=\frac{7}{2}, \quad E=\frac{28}{3}, \quad N_{\mathrm{DW}}=7,
\end{aligned}
$$

for two models. The leading gauge-invariant PQ-breaking operators become ${ }^{2}$

$$
\begin{aligned}
& \mathrm{SU}(7)-\mathrm{A} \quad: \quad \mathcal{O}_{\Delta \mathrm{PQ}=-8}^{d=10}=\left(\overline{\mathbf{2}}_{\mathbf{H}}\right)^{8} \cdot \mathbf{7}_{\mathbf{H}}{ }^{2}=\epsilon^{A_{1} \ldots A_{7}} \epsilon^{B_{1} \ldots B_{7}} \epsilon^{\rho_{1} \ldots \rho_{8}}
\end{aligned}
$$

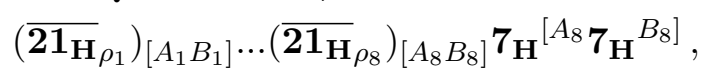

$$
\begin{aligned}
& \mathrm{SU}(7)-\mathrm{B} \quad: \quad \mathcal{O}_{\Delta \mathrm{PQ}=-7}^{d=7}=\left({\overline{\mathbf{7}_{\mathbf{H}}}}_{\rho}\right)^{7}
\end{aligned}
$$

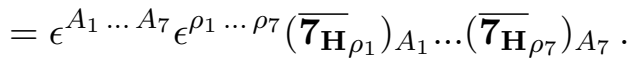

For the leading PQ-breaking operator in the SU(7)-A model, its mass dimension of 10 can guarantee the PQ-quality constraint even if all Higgs fields of $\overline{\mathbf{2 1}}_{\mathbf{H}} \rho$ and $\mathbf{7}_{\mathbf{H}}$ develop their VEVs at $\sim f_{a}$. For the $\mathrm{SU}(7)$-B model, the leading PQ-breaking operator is of mass dimension 7 . In order to have a consistent axion decay constant of $f_{a} \gtrsim 10^{8} \mathrm{GeV}$, it is necessary that one of the $\overline{\mathbf{7}_{\mathbf{H}} \rho}$ develops its VEV for the electroweak symmetry breaking (EWSB).

\section{B. The $\mathrm{SU}(8)$}

For the $\mathrm{SU}(8)$ group, the anomaly vector in Eq. 13 reads

$$
\vec{A}=(0,1,4,5,0,-5,-4,-1) .
$$

The decompositions of the SU(8) irreps under the SU(5) are the following

$$
\begin{aligned}
\mathbf{8} & =[8,1]=3 \times[5,0] \oplus[5,1], \\
\mathbf{2 8} & =[8,2]=3 \times[5,0] \oplus 3 \times[5,1] \oplus[5,2], \\
\mathbf{5 6} & =[8,3]=[5,0] \oplus 3 \times[5,1] \oplus 3 \times[5,2] \oplus[5,3] .
\end{aligned}
$$

The possibility for $n_{g}=3$ with the minimal anomaly-free fermion content is given by

$$
\begin{aligned}
\left\{f_{L}\right\}_{\mathrm{SU}(8)} & =[8,2] \oplus[8,3] \oplus 9 \times[8,7]=\mathbf{2 8}_{\mathbf{F}} \oplus \mathbf{5 6}_{\mathbf{F}} \oplus 9 \times \overline{\mathbf{8}_{\mathbf{F}}}, \quad \operatorname{dim}_{\mathbf{F}}=156, \\
\mathcal{G}_{\text {global }} & =\mathrm{SU}(9)_{\overline{\mathbf{8}_{\mathbf{F}}}} \otimes \mathrm{U}(1)_{\mathrm{PQ}} .
\end{aligned}
$$

\footnotetext{
${ }^{2}$ Throughout the context, we use the square brackets to anti-symmetrize the indices.
} 


\begin{tabular}{c|ccc|ccccc}
\hline & $9 \times \overline{\mathbf{8}_{\mathbf{F}}}$ & $\mathbf{2 8}_{\mathbf{F}}$ & $\mathbf{5 6} \mathbf{6}_{\mathbf{F}}$ & $9 \times \overline{\mathbf{8}_{\mathbf{H}}}$ & $9 \times \overline{\mathbf{2 8}_{\mathbf{H}}}$ & $\mathbf{2 8}_{\mathbf{H}}$ & $\mathbf{5 6}_{\mathbf{H}}$ & $\mathbf{7 0}_{\mathbf{H}}$ \\
\hline PQ charges & 1 & 0 & 0 & -1 & 0 & 0 & 0 & 0 \\
$\mathrm{SU}(9)_{\overline{\mathbf{8}_{\mathbf{F}}}}$ & $\square$ & 1 & 1 & $\overline{0}$ & 1 & 1 & 1 & 1 \\
\hline
\end{tabular}

Table II. The PQ charge assignments and the $\mathrm{SU}(9)_{F}$ representations of the fermions and Higgs fields in the minimal SU(8) unification model.

The Higgs sector of the $\mathrm{SU}(8)$ is determined by the fermions and the global symmetries in Eq. (33) as follows ${ }^{3}$

$$
\{H\}_{\mathrm{SU}(8)}=9 \times \overline{\mathbf{8}_{\mathbf{H}}} \oplus 9 \times \overline{\mathbf{2 8}_{\mathbf{H}}} \oplus \mathbf{2} \mathbf{8}_{\mathbf{H}} \oplus \mathbf{5 6}_{\mathbf{H}} \oplus \mathbf{7 0}_{\mathbf{H}}\left[\oplus \mathbf{6 3}_{\mathbf{H}}\right] .
$$

By using the fermions and Higgs fields in Eqs. (33) and (34), we find that $b_{1}=-\frac{2}{3}<0$. Thus the minimal SU(8) model is asymptotically free. The gauge-invariant Yukawa couplings are

$$
\begin{aligned}
-\mathcal{L}_{Y} & =\sum_{\rho=1}^{9}\left({\overline{\mathbf{8}_{\mathbf{F}}}}^{\rho} \mathbf{2 8 _ { \mathbf { F } }}{\overline{\mathbf{8}_{\mathbf{H}}}}_{\rho}+{\overline{\mathbf{8}_{\mathbf{F}}}}^{\rho} \mathbf{5} \mathbf{6}_{\mathbf{F}} \overline{\mathbf{2 8}}_{\mathbf{H}} \rho\right) \\
& +\mathbf{5 6}_{\mathbf{F}} \mathbf{5 6 _ { \mathbf { F } }} \mathbf{2 8 _ { \mathbf { H } }}+\mathbf{2 8}_{\mathbf{F}} \mathbf{5 6} \mathbf{6}_{\mathbf{F}} \mathbf{5} \mathbf{6}_{\mathbf{H}}+\mathbf{2 8}_{\mathbf{F}} \mathbf{2 8 _ { \mathbf { F } }} \mathbf{7 0}_{\mathbf{H}}+\text { H.c. }
\end{aligned}
$$

We assign PQ charges for all SU(8) fields in Tab. II, with the same argument in the $\mathrm{SU}(7)$ models. The color and electromagnetic anomaly factors, and domain wall numbers according to Eq. (22) are given by

$$
\mathrm{SU}(8): N_{\mathrm{SU}(3)_{c}}=\frac{9}{2}, \quad E=12, \quad N_{\mathrm{DW}}=9 .
$$

The leading PQ-breaking operators in the SU(8) model is

$$
\begin{aligned}
\mathrm{SU}(8): & \mathcal{O}_{\Delta \mathrm{PQ}=-9}^{d=12}=\left(\overline{\mathbf{8}_{\mathbf{H} \rho}}\right)^{9} \cdot \mathbf{2 8}_{\mathbf{H}} \cdot \mathbf{5} \mathbf{6}_{\mathbf{H}} \cdot \mathbf{7 0}_{\mathbf{H}} \\
= & \epsilon^{A_{1} \ldots A_{8}} \epsilon_{B_{1} \ldots B_{8}} \epsilon^{\rho_{1} \ldots \rho_{8} \delta}\left(\overline{\mathbf{8}_{\mathbf{H}}} \rho_{1}\right)_{\left.A_{1} \ldots\left(\overline{\mathbf{8}}_{\mathbf{H}}\right)_{8}\right)_{A_{8}}\left(\overline{\mathbf{8}_{\mathbf{H}}}\right)_{B_{0}}} \\
& \left(\mathbf{2 8}_{\mathbf{H}}\right)^{\left[B_{0} B_{1}\right]}\left(\mathbf{5 6}_{\mathbf{H}}\right)^{\left[B_{2} B_{3} B_{4}\right]}\left(\mathbf{7 0}_{\mathbf{H}}\right)^{\left[B_{5} B_{6} B_{7} B_{8}\right]} .
\end{aligned}
$$

C. The $\mathrm{SU}(9)$

For the SU(9) group, the anomaly vector in Eq. (13) reads

$$
\vec{A}=(0,1,5,9,5,-5,-9,-5,-1) \text {. }
$$

\footnotetext{
${ }^{3}$ In the $\mathrm{SU}(8)$, the 70 -dimensional irrep is self-conjugate.
} 
The decompositions of the $\mathrm{SU}(9)$ irreps under the $\mathrm{SU}(5)$ are the following

$$
\begin{aligned}
\mathbf{9} & =[9,1]=4 \times[5,0] \oplus[5,1] \\
\mathbf{3 6} & =[9,2]=6 \times[5,0] \oplus 4 \times[5,1] \oplus[5,2] \\
\mathbf{8 4} & =[9,3]=4 \times[5,0] \oplus 6 \times[5,1] \oplus 4 \times[5,2] \oplus[5,3] \\
\mathbf{1 2 6} & =[9,4]=[5,0] \oplus 4 \times[5,1] \oplus 6 \times[5,2] \oplus 4 \times[5,3] \oplus[5,4] .
\end{aligned}
$$

The possibility for $n_{g}=3$ with the minimal anomaly-free fermion content is given by

$$
\begin{aligned}
\left\{f_{L}\right\}_{\mathrm{SU}(9)} & =[9,3] \oplus 9 \times[9,8]=\mathbf{8 4}_{\mathbf{F}} \oplus 9 \times \overline{\mathbf{9}_{\mathbf{F}}}, \quad \operatorname{dim}_{\mathbf{F}}=165 \\
\mathcal{G}_{\text {global }} & =\mathrm{SU}(9)_{\overline{\mathbf{9}_{\mathbf{F}}}} \otimes \mathrm{U}(1)_{\mathrm{PQ}} .
\end{aligned}
$$

Another possibility with more fermions of $[9,2] \oplus[9,4] \oplus 10 \times[9,8]$ will not be considered here. An even larger fermion contents of $2 \times[9,2] \oplus 2 \times[9,4] \oplus[9,6] \oplus 11 \times[9,8]$ were previously mentioned in Ref. [5].

\begin{tabular}{c|cc|cc}
\hline & $9 \times \overline{\mathbf{9}_{\mathbf{F}}}$ & $\mathbf{8 4}$ & $9 \times \overline{\mathbf{3 6}}$ & $\mathbf{8 4}_{\mathbf{H}}$ \\
\hline PQ charges & 1 & 0 & -1 & 0 \\
SU(9) $\overline{\mathbf{9}_{\mathbf{F}}}$ & $\square$ & 1 & $\square$ & 1 \\
\hline
\end{tabular}

Table III. The PQ charge assignments and the $\mathrm{SU}(9)_{F}$ representations of the fermions and Higgs fields in the minimal SU(9) unification model.

The Higgs sector of the SU(9) is determined by the fermions and the global symmetries in Eq. 40 as follows

$$
\{H\}_{\mathrm{SU}(9)}=9 \times \overline{\mathbf{3 6}_{\mathbf{H}}} \oplus \mathbf{8 4}_{\mathbf{H}}\left[\oplus \mathbf{8 0}_{\mathbf{H}}\right] .
$$

By using the fermions and Higgs fields in Eqs. 40) and 41], we find that $b_{1}=-\frac{15}{2}<0$. Thus the minimal SU(9) model is asymptotically free. The gauge-invariant Yukawa couplings are

$$
-\mathcal{L}_{Y}={\overline{\mathbf{9}_{\mathbf{F}}}}^{\rho} \mathbf{8 4 _ { \mathbf { F } }} \overline{\mathbf{3 6}}_{\mathbf{H}}+\mathbf{8 4}_{\mathbf{F}} \mathbf{8} 4_{\mathbf{F}} \mathbf{8} 4_{\mathbf{H}}+\text { H.c. },
$$

and we assign the PQ charges in Tab. III. Naively, the PQ charge of the $\mathbf{8 4}_{\mathbf{H}}$ can be arbitrary according to the Yukawa couplings 42 . It turns out a dimension-3 PQ-breaking operator of $\mathcal{O}_{\mathrm{RQ}}^{d=3}=\left(\mathbf{8 4}_{\mathbf{H}}\right)^{3}=\epsilon_{A_{1} B_{1} C_{1} A_{2} B_{2} C_{2} A_{3} B_{3} C_{3}}\left(\mathbf{8 4}_{\mathbf{H}}\right)^{\left[A_{1} B_{1} C_{1}\right]}\left(\mathbf{8 4}_{\mathbf{H}}\right)^{\left[A_{2} B_{2} C_{2}\right]}\left(\mathbf{8 4}_{\mathbf{H}}\right)^{\left[A_{3} B_{3} C_{3}\right]}$ can arise, which is dangerous from the dimensional counting. Therefore, we determine 
that $\mathrm{PQ}\left(\mathbf{8 4}_{\mathbf{H}}\right)=0$. The color and electromagnetic anomaly factors, and domain wall numbers according to Eq. 22 ) are given by

$$
\mathrm{SU}(9): N_{\mathrm{SU}(3)_{c}}=\frac{9}{2}, \quad E=12, \quad N_{\mathrm{DW}}=9 .
$$

The leading dimension-9 PQ-breaking operator in the SU(9) model is

$$
\mathcal{O}_{\Delta \mathrm{PQ}=-9}^{d=9}=\left(\overline{\mathbf{3 6}_{\mathbf{H}}}\right)^{9}=\epsilon^{A_{1} \ldots A_{9}} \epsilon^{B_{1} \ldots B_{9}} \epsilon^{\rho_{1} \ldots \rho_{9}}\left(\overline{\mathbf{3 6}}_{\mathbf{H}}^{\rho_{1}}\right)_{\left[A_{1} B_{1}\right]} \ldots\left(\overline{\mathbf{3 6}}_{\mathbf{H}} \rho_{9}\right)_{\left[A_{9} B 9\right]} .
$$

According to the dimension counting in Ref. [27, this is likely to produce a high-quality axion.

\section{The $\mathrm{SU}(10)$}

For the SU(10) group, the anomaly vector in Eq. 13 reads

$$
\vec{A}=(0,1,6,14,14,0,-14,-14,-6,-1) .
$$

The decompositions of the SU(10) irreps under the SU(5) are the following

$$
\begin{aligned}
\mathbf{1 0} & =[10,1]=5 \times[5,0] \oplus[5,1], \\
\mathbf{4 5} & =[10,2]=10 \times[5,0] \oplus 5 \times[5,1] \oplus[5,2], \\
\mathbf{1 2 0} & =[10,3]=10 \times[5,0] \oplus 10 \times[5,1] \oplus 5 \times[5,2] \oplus[5,3], \\
\mathbf{2 1 0} & =[10,4]=5 \times[5,0] \oplus 10 \times[5,1] \oplus 10 \times[5,2] \oplus 5 \times[5,3] \oplus[5,4] .
\end{aligned}
$$

The possibility for $n_{g}=3$ with the minimal anomaly-free fermion content is given by

$$
\begin{aligned}
\left\{f_{L}\right\}_{\mathrm{SU}(10)} & =[10,3] \oplus[10,8] \oplus 8 \times[10,9] \\
& =\mathbf{1 2 0}_{\mathbf{F}} \oplus \overline{\mathbf{4 5}_{\mathbf{F}}} \oplus 8 \times \overline{\mathbf{1 0}_{\mathbf{F}}}, \quad \operatorname{dim}_{\mathbf{F}}=245, \\
\mathcal{G}_{\text {global }} & =\mathrm{SU}(8)_{\overline{\mathbf{1 0}_{\mathbf{F}}}} \otimes \mathrm{U}(1)_{\mathrm{PQ}} .
\end{aligned}
$$

\begin{tabular}{c|ccc|cccc}
\hline & $8 \times \overline{\mathbf{1 0}_{\mathbf{F}}} \overline{\mathbf{4 5}_{\mathbf{F}}}$ & $\mathbf{1 2 0}_{\mathbf{F}}$ & $8 \times \overline{\mathbf{4 5}_{\mathbf{H}}}$ & $8 \times \mathbf{1 2 0}_{\mathbf{H}}$ & $\overline{\mathbf{1 0}_{\mathbf{H}}}$ & $\mathbf{2 1 0}_{\mathbf{H}}$ \\
\hline PQ charges & 1 & 0 & 0 & -1 & -1 & 0 & 0 \\
$\mathrm{SU}(8) \overline{\mathbf{1 0}_{\mathbf{F}}}$ & $\square$ & 1 & 1 & $\square$ & $\square$ & 1 & 1 \\
\hline
\end{tabular}

Table IV. The PQ charge assignments and the $\mathrm{SU}(8) \overline{\mathbf{1 0}_{\mathrm{F}}}$ representations of the fermions and Higgs fields in the minimal SU(10) unification model. 
The Higgs sector of the SU(10) is determined by the fermions and the global symmetries in Eq. (47) as follows

$$
\{H\}_{\mathrm{SU}(10)}=8 \times \overline{\mathbf{4 5}} \oplus 8 \times \mathbf{1 2 0}_{\mathbf{H}} \oplus \overline{\mathbf{1 0}_{\mathbf{H}}} \oplus \mathbf{2 1 0 _ { \mathbf { H } }}\left[\oplus \mathbf{9 9}_{\mathbf{H}}\right] .
$$

By using the fermions and Higgs fields in Eqs. 47) and (48), we find that $b_{1}=\frac{223}{6}$. Thus the minimal SU(10) model is not asymptotically free. The gauge-invariant Yukawa couplings are

$$
\begin{aligned}
-\mathcal{L}_{Y} & ={\overline{10_{\mathbf{F}}}}^{\rho} 120_{\mathbf{F}}{\overline{45_{\mathbf{H}}}}_{\rho}+{\overline{10_{\mathbf{F}}}}^{\rho} \overline{4_{\mathbf{F}}} 120_{\mathbf{H} \rho} \\
& +\left(\overline{45_{\mathbf{F}} 45_{\mathbf{F}}}+120_{\mathbf{F}} 120_{\mathbf{F}}\right) \mathbf{2 1 0}_{\mathbf{H}}+\overline{45_{\mathbf{F}}} 120_{\mathbf{F}} \overline{10_{\mathbf{H}}}+\text { H.c. }
\end{aligned}
$$

and we assign the PQ charges in Tab. IV] It turns out that three dimension-5 PQbreaking operators of $\mathcal{O}_{\mathrm{RQ}}^{d=5}=\left(\overline{\mathbf{1 0}_{\mathbf{H}}}\right)^{4} \mathbf{2 1 0}_{\mathbf{H}}, \mathcal{O}_{\mathrm{RQ}}^{d=5}=\left(\overline{\mathbf{1 0}_{\mathbf{H}}}\right)^{2}\left(\mathbf{2 1 0}_{\mathbf{H}}\right)^{3}$, and $\mathcal{O}_{\mathrm{RQ}}^{d=5}=$ $\left(\mathbf{2 1 0}_{\mathbf{H}}\right)^{5}$ can arise if $\mathrm{PQ}\left(\overline{\mathbf{1 0}_{\mathbf{H}}}\right) \neq 0$ or $\mathrm{PQ}\left(\mathbf{2 1 0}_{\mathbf{H}}\right) \neq 0$. Therefore, we determine that $\mathrm{PQ}(\overline{\mathbf{1 0}})=0$ and $\mathrm{PQ}\left(\mathbf{2 1 0}_{\mathbf{H}}\right)=0$. The color and electromagnetic anomaly factor, and domain wall numbers according to Eq. (22) are given by

$$
\mathrm{SU}(10): N_{\mathrm{SU}(3)_{c}}=4, \quad E=\frac{32}{3}, \quad N_{\mathrm{DW}}=8 .
$$

Two dimension-12 PQ-breaking operators in the SU(10) model are expressed as follows

$$
\begin{aligned}
& \mathcal{O}_{\Delta \mathrm{PQ}=-8}^{d=12}=(\overline{\mathbf{4 5}})^{8}\left(\overline{\mathbf{1 0}_{\mathbf{H}}}\right)^{2}\left(\mathbf{2 1 0}_{\mathbf{H}}\right)^{2} \\
& =\epsilon^{A_{1} B_{1} \ldots A_{5} B_{5}} \epsilon^{\rho_{1} \ldots \rho_{8}}\left(\overline{\mathbf{4 5}}_{\mathbf{H}} \rho_{1}\right)\left[A_{1} B_{1}\right] \ldots\left(\overline{\mathbf{4 5}}_{\mathbf{H}} \rho_{5}\right)_{\left[A_{5} B_{5}\right]} \\
& \left(\overline{\mathbf{4 5}}_{\rho_{6}}\right)_{\left[A_{6} B_{6}\right]}\left(\overline{\mathbf{4 5}}_{\mathbf{H}_{7}}\right)_{\left[A_{7} B_{7}\right]}\left(\overline{\mathbf{4 5}_{\mathbf{H}}} \rho_{8}\right)_{\left[A_{8} B_{8}\right]}\left(\overline{\mathbf{1 0}_{\mathbf{H}}}\right)_{C_{1}}\left(\overline{\mathbf{1 0}_{\mathbf{H}}}\right)_{C_{2}} \\
& \left(\mathbf{2 1 0}_{\mathbf{H}}\right)^{\left[A_{6} A_{7} A_{8} C_{1}\right]}\left(\mathbf{2 1 0}_{\mathbf{H}}\right)^{\left[B_{6} B_{7} B_{8} C_{2}\right]} \text {, } \\
& \mathcal{O}_{\triangle \mathrm{PQ}=-8}^{d=12}=\left(\mathbf{1 2 0}_{\mathbf{H}}\right)^{8}\left(\overline{\mathbf{1 0}_{\mathbf{H}}}\right)^{2}\left(\mathbf{2 1 0}_{\mathbf{H}}\right)^{2} \\
& =\epsilon^{A_{1} \ldots A_{8} A_{9} A_{10}} \epsilon^{B_{1} \ldots B_{8} B_{9} B_{10}} \epsilon^{C_{1} \ldots C_{8} C_{9} C_{10}} \epsilon^{\rho_{1} \ldots \rho_{8}} \\
& \left(\mathbf{1 2 0}_{\mathbf{H}} \rho_{1}\right)^{\left[A_{1} B_{1} C_{1}\right]} \ldots\left(\mathbf{1 2 0}_{\mathbf{H} \rho_{8}}\right)^{\left[A_{8} B_{8} C_{8}\right]}\left(\overline{\mathbf{1 0}_{\mathbf{H}}}\right)_{D_{1}}\left(\overline{\mathbf{1 0}_{\mathbf{H}}}\right)_{D_{2}} \\
& \left(\mathbf{2 1 0}_{\mathbf{H}}\right)^{\left[D_{1} A_{9} B_{9} C_{9}\right]}\left(\mathbf{2 1 0}_{\mathbf{H}}\right)^{\left[D_{2} A_{10} B_{10} C_{10}\right]} \text {. }
\end{aligned}
$$

E. The $\mathrm{SU}(11)$

For the $\mathrm{SU}(11)$ group, the anomaly vector in Eq. 13 reads

$$
\vec{A}=(0,1,7,20,28,14,-14,-28,-20,-7,-1) \text {. }
$$


The decompositions of the SU(11) irreps under the $\mathrm{SU}(5)$ are the following

$$
\begin{aligned}
\mathbf{1 1} & =[11,1]=6 \times[5,0] \oplus[5,1], \\
\mathbf{5 5} & =[11,2]=15 \times[5,0] \oplus 6 \times[5,1] \oplus[5,2], \\
\mathbf{1 6 5} & =[11,3]=20 \times[5,0] \oplus 15 \times[5,1] \oplus 6 \times[5,2] \oplus[5,3], \\
\mathbf{3 3 0} & =[11,4]=15 \times[5,0] \oplus 20 \times[5,1] \oplus 15 \times[5,2] \oplus 6 \times[5,3] \oplus[5,4], \\
\mathbf{4 6 2} & =[11,5]=7 \times[5,0] \oplus 15 \times[5,1] \oplus 20 \times[5,2] \oplus 15 \times[5,3] \oplus 6 \times[5,4] .
\end{aligned}
$$

\begin{tabular}{|c|c|c|c|c|c|c|c|}
\hline & $6 \times \overline{\mathbf{1 1}_{\mathbf{F}}}$ & $2 \times \overline{\mathbf{5 5}}$ & $165_{F}$ & $6 \times \overline{\mathbf{5 5}_{\mathbf{H}}}$ & $2 \times \overline{\mathbf{1 1}_{\mathbf{H}}}$ & $330_{\mathrm{H}}$ & $462_{\mathrm{H}}$ \\
\hline PQ charges & 1 & 0 & 0 & -1 & -1 & 0 & 0 \\
\hline $\mathrm{SU}(6)_{\overline{\mathbf{1 1}_{\mathbf{F}}}}$ & $\square$ & 1 & 1 & $\bar{\square}$ & $\bar{\square}$ & 1 & 1 \\
\hline
\end{tabular}

The possibility for $n_{g}=3$ with the minimal anomaly-free fermion content is given by

$$
\begin{aligned}
\left\{f_{L}\right\}_{\mathrm{SU}(11)} & =[11,3] \oplus 2 \times[11,9] \oplus 6 \times[11,10] \\
& =\mathbf{1 6 5}_{\mathbf{F}} \oplus 2 \times \overline{\mathbf{5 5}} \oplus 6 \times \overline{\mathbf{1 1}}, \quad \operatorname{dim}_{\mathbf{F}}=341 \\
\mathcal{G}_{\text {global }} & =\left[\mathrm{SU}(6)_{\overline{\mathbf{1 1}_{\mathbf{F}}}} \otimes \mathrm{U}(1)_{\mathrm{PQ}}\right] \otimes\left[\mathrm{SU}(2) \otimes \mathrm{U}(1)^{\prime}\right] .
\end{aligned}
$$

Table V. The PQ charge assignments and the $\mathrm{SU}(6)_{\overline{\mathbf{1}} \mathbf{F}}$ representations of the fermions and Higgs fields in the minimal SU(11) unification model.

The Higgs sector of the SU(11) is determined by the fermions and the global symmetries in Eq. (54) as follows

$$
\{H\}_{\mathrm{SU}(11)}=6 \times \overline{\mathbf{5 5}_{\mathbf{H}}} \oplus 2 \times \overline{\mathbf{1 1}_{\mathbf{H}}} \oplus \mathbf{3 3 0 _ { \mathbf { H } }} \oplus \mathbf{4 6 2} \mathbf{H}[\oplus \mathbf{1 2 0} \mathbf{H}] .
$$

By using the fermions and Higgs fields in Eqs. (54) and (55), we find that $b_{1}=\frac{83}{3}$. The gauge-invariant Yukawa couplings are

$$
\begin{aligned}
-\mathcal{L}_{Y} & =\sum_{\rho=1}^{6} \overline{\mathbf{1 1}}_{\mathbf{F}}^{\rho} \mathbf{1 6 5}_{\mathbf{F}} \overline{\mathbf{5 5}}_{\mathbf{H}}+\sum_{\dot{\rho}=1,2} \overline{\mathbf{5 5}}_{\mathbf{F}} \dot{ } \mathbf{1 6 5}_{\mathbf{F}} \overline{\mathbf{1 1}}_{\mathbf{H}} \dot{\rho} \\
& +\epsilon_{\dot{\rho} \sigma} \overline{\mathbf{5 5}}_{\mathbf{F}}^{\dot{\rho}} \overline{\mathbf{5 5}}_{\mathbf{F}}^{\dot{\sigma}} \mathbf{3 3 0} \mathbf{H}_{\mathbf{H}}+\mathbf{1 6 5}_{\mathbf{F}} \mathbf{1 6 5 _ { \mathbf { F } }} \mathbf{4 6 2} \mathbf{H}+\text { H.c. }
\end{aligned}
$$

We assign the PQ charges in Tab. V. It turns out a possible dimension-5 PQ-breaking operator of $\mathcal{O}_{\mathrm{RQ}}^{d=5}=\left(\mathbf{3 3 0}_{\mathbf{H}}\right)^{3}\left(\mathbf{4 6 2}_{\mathbf{H}}\right)^{2}$ may arise. For this reason we consider $\mathrm{PQ}\left(\mathbf{3 3 0}_{\mathbf{H}}\right)=0$ and $\mathrm{PQ}\left(\mathbf{4 6 2}_{\mathbf{H}}\right)=0$. The color and electromagnetic anomaly factor, and domain wall numbers according to Eq. 222 are given by

$$
\mathrm{SU}(11): N_{\mathrm{SU}(3)_{c}}=3, \quad E=8, \quad N_{\mathrm{DW}}=6 .
$$


The leading PQ-breaking operators in the SU(11) model is found to be

$$
\begin{aligned}
\mathcal{O}_{\mathrm{RQ}}^{d=9}= & \left(\overline{\mathbf{5 5}_{\mathbf{H}}}\right)^{6}\left(\mathbf{3 3 0}_{\mathbf{H}}\right)^{3} \\
= & \epsilon_{A_{1} B_{1} \ldots A_{6}} \epsilon^{\rho_{1} \ldots \rho_{6}}\left(\overline{\mathbf{5}} \mathbf{H}_{\rho_{1}}\right)_{\left[A_{1} B_{1}\right] \ldots\left(\overline{\mathbf{5 5}_{\mathbf{H}}} \rho_{6}\right)_{\left[A_{6} C\right]}} \\
& \epsilon_{D_{1} E_{1} \ldots E_{3} F_{3}}\left(\mathbf{3 3 0}_{\mathbf{H}}\right)^{\left[D_{1} E_{1} F_{1} G_{1}\right]}\left(\mathbf{3 3 0}_{\mathbf{H}}\right)^{\left[D_{2} E_{2} F_{2} G_{2}\right]}\left(\mathbf{3 3 0}_{\mathbf{H}}\right)^{\left[D_{3} E_{3} F_{3} C\right]} .
\end{aligned}
$$

\begin{tabular}{|c|c|c|c|c|}
\hline Models & $\operatorname{dim}_{F}$ & $b_{1}$ & $N_{\mathrm{SU}(3)_{\mathrm{c}}}$ & PQ-breaking \\
\hline $\mathrm{SU}(7)-\mathrm{A}$ & 133 & -5 & 4 & $\left(\overline{\mathbf{2}}_{\mathbf{H}}\right)^{8} \cdot\left(\mathbf{7}_{\mathbf{H}}\right)^{2}$ \\
\hline $\mathrm{SU}(7)-\mathrm{B}$ & 140 & $-\frac{55}{6}$ & $7 / 2$ & $\left(\overline{\mathbf{7}}_{\mathbf{H}}\right)^{7}$ \\
\hline $\mathrm{SU}(8)$ & 156 & $-\frac{2}{3}$ & $9 / 2$ & $\left(\overline{\mathbf{8}_{\mathbf{H} \rho}}\right)^{9} \cdot \mathbf{2} \mathbf{8}_{\mathbf{H}} \cdot \mathbf{5 6} \mathbf{6}_{\mathbf{H}} \cdot \mathbf{7 0}_{\mathbf{H}}$ \\
\hline $\mathrm{SU}(9)$ & 165 & $-\frac{15}{2}$ & $9 / 2$ & $\left(\overline{\mathbf{3 6}_{\mathbf{H}}}\right)^{9}$ \\
\hline \multirow[t]{2}{*}{$\mathrm{SU}(10)$} & 245 & $\frac{223}{6}$ & 4 & $(\overline{\mathbf{4 5}})^{8}\left(\overline{\mathbf{1 0}_{\mathbf{H}}}\right)^{2}\left(\mathbf{2 1 0 _ { \mathbf { H } }}\right)^{2}$ \\
\hline & & & & $\left(120_{\mathbf{H}}\right)^{8}\left(\overline{10_{\mathbf{H}}}\right)^{2}\left(210_{\mathbf{H}}\right)^{2}$ \\
\hline $\mathrm{SU}(11)$ & 341 & $\frac{83}{3}$ & 3 & $\left(\overline{55_{\mathbf{H}}}\right)^{6}\left(\mathbf{3 3 0}_{\mathbf{H}}\right)^{3}$ \\
\hline
\end{tabular}

\section{CONCLUSIONS}

Table VI. The non-minimal GUTs with $n_{g}=3$, their one-loop $\beta$ coefficients, the color anomaly factors, and the PQ-breaking operators.

We have studied the set of non-minimal GUTs that can lead to the observed three generational SM fermions according to Georgi's counting. With the origin of the generational structure, these models themselves can be appealing to answer the most puzzling question of the SM fermion mass hierarchies. Our results suggest four such models that achieve the AF property at short distances, and two more that may be considered with further studies. The results are summarized in Tab. VI. The other important feature of the non-minimal GUTs in our study comes from their global symmetries, which was also previously noted in the SU(6) model [23, 31]. Though the SU(6) model enjoys a global DRS symmetry, it turns out the SUSY extension was inevitable in order to produce a high-quality PQ symmetry. In six non-minimal GUTs of the current study, the sizes of the PQ-breaking effects due to the quantum gravity are generally under better control due to the gauge symmetries and the associated global DRS symmetries. It is thus reasonable to expect the long-standing PQ quality problem can be avoided in non-minimal GUTs with $n_{g}=3$, where the emergent global DRS symmetries are general. 
Obviously, we expect the following studies to be performed for specific models, which include: (i) the viable symmetry breaking patterns, (ii) SM fermion mass hierarchies and their mixings, (iii) the physical axion mass predictions and the related experimental searches. A recent study of the $\mathrm{SU}(6)$ toy model [39] suggests that the bottom quark and tau lepton masses can be naturally suppressed to the top quark mass through the seesawlike mass matrices with their heavy fermion partners. Such heavy fermion partners for both the down-type quarks and charged leptons are general in the non-minimal GUTs with $n_{g}=3$. Furthermore, the non-minimal GUTs with $n_{g}=3$ can naturally lead to multiple symmetry breaking scales between the $\Lambda_{\mathrm{GUT}}$ and the EW scale. Altogether, we expect that the observed fermion mass hierarchies among three generations can be realized with the appropriate symmetry breaking pattern in the non-minimal GUT with $n_{g}=3$. Above all, one has to analyze the gauge coupling unifications for the viable symmetry breaking patterns and predicts the proton lifetime. Since our models possess several intermediate scales, this usually requires the two-loop RGEs, together with the matching conditions and mass threshold effects [49, 50].

\section{ACKNOWLEDGEMENTS}

We would like to thank Luca Di Luzio, Jing Ren, and Ye-Ling Zhou for very useful discussions and communication. N.C. would like to thank Tibet University for hospitality when preparing this work. This work is partially supported by the National Natural Science Foundation of China (under Grant No. 12035008 and No. 11575176).

[1] H. Georgi and S. L. Glashow, Phys. Rev. Lett. 32, 438 (1974).

[2] H. Fritzsch and P. Minkowski, Annals Phys. 93, 193 (1975).

[3] S. Dimopoulos and H. Georgi, Nucl. Phys. B 193, 150 (1981).

[4] H. Georgi and C. Jarlskog, Phys. Lett. B 86, 297 (1979).

[5] S. M. Barr, Phys. Rev. D 21, 1424 (1980).

[6] H. Georgi and D. V. Nanopoulos, Nucl. Phys. B 159, 16 (1979).

[7] H. Georgi, Fermion Masses in Unified Theories, in First Workshop on Grand Unification, 1980.

[8] S. Dimopoulos, L. J. Hall, and S. Raby, Phys. Rev. Lett. 68, 1984 (1992).

[9] G. F. Giudice, Mod. Phys. Lett. A 7, 2429 (1992), hep-ph/9204215. 
[10] P. Ramond, R. G. Roberts, and G. G. Ross, Nucl. Phys. B 406, 19 (1993), hep-ph/9303320.

[11] L. J. Hall, R. Rattazzi, and U. Sarid, Phys. Rev. D 50, 7048 (1994), hep-ph/9306309.

[12] L. E. Ibanez and G. G. Ross, Phys. Lett. B 332, 100 (1994), hep-ph/9403338.

[13] P. Nath, Phys. Rev. Lett. 76, 2218 (1996), hep-ph/9512415.

[14] S. M. Barr, Phys. Rev. D 76, 105024 (2007), 0706.1490.

[15] H. Georgi, Nucl. Phys. B 156, 126 (1979).

[16] P. H. Frampton, Phys. Lett. B 88, 299 (1979).

[17] P. H. Frampton, P. Q. Hung, and M. Sher, Phys. Rept. 330, 263 (2000), hep-ph/9903387.

[18] ATLAS, G. Aad et al., Phys. Rev. Lett. 108, 261802 (2012), 1202.3076.

[19] ATLAS, G. Aad et al., Phys. Rev. Lett. 109, 032001 (2012), 1202.6540.

[20] CMS, S. Chatrchyan et al., JHEP 05, 123 (2012), 1204.1088.

[21] N. D. Christensen and R. Shrock, Phys. Rev. D 72, 035013 (2005), hep-ph/0506155

[22] H. M. Georgi, L. J. Hall, and M. B. Wise, Nucl. Phys. B 192, 409 (1981).

[23] G. R. Dvali, Phys. Lett. B 324, 59 (1994).

[24] R. D. Peccei and H. R. Quinn, Phys. Rev. Lett. 38, 1440 (1977).

[25] M. Dine and N. Seiberg, Nucl. Phys. B 273, 109 (1986).

[26] S. M. Barr and D. Seckel, Phys. Rev. D 46, 539 (1992).

[27] M. Kamionkowski and J. March-Russell, Phys. Lett. B 282, 137 (1992), hep-th/9202003

[28] R. Holman et al., Phys. Lett. B 282, 132 (1992), hep-ph/9203206.

[29] S. Ghigna, M. Lusignoli, and M. Roncadelli, Phys. Lett. B 283, 278 (1992).

[30] L. Di Luzio, JHEP 11, 074 (2020), 2008.09119.

[31] N. Chen, Y. Liu, and Z. Teng, Phys. Rev. D 104, 115011 (2021), 2106.00223.

[32] R. Feger, T. W. Kephart, and R. J. Saskowski, Comput. Phys. Commun. 257, 107490 (2020), 1912.10969.

[33] S. Dimopoulos, S. Raby, and L. Susskind, Nucl. Phys. B 173, 208 (1980).

[34] J. Banks and H. Georgi, Phys. Rev. D 14, 1159 (1976).

[35] S. Okubo, Phys. Rev. D 16, 3528 (1977).

[36] L.-F. Li, Phys. Rev. D 9, 1723 (1974).

[37] Super-Kamiokande, A. Takenaka et al., Phys. Rev. D 102, 112011 (2020), 2010.16098.

[38] T. Li, J. Pei, F. Xu, and W. Zhang, Phys. Rev. D 102, 016004 (2020), 1911.09551.

[39] N. Chen, Y.-n. Mao, and Z. Teng, (2021), 2112.14509.

[40] D. F. Litim and F. Sannino, JHEP 12, 178 (2014), 1406.2337.

[41] D. F. Litim, M. Mojaza, and F. Sannino, JHEP 01, 081 (2016), 1501.03061. 
[42] J. E. Kim, Phys. Rev. D 21, 1687 (1980).

[43] M. B. Wise, H. Georgi, and S. L. Glashow, Phys. Rev. Lett. 47, 402 (1981).

[44] L. Di Luzio, A. Ringwald, and C. Tamarit, Phys. Rev. D 98, 095011 (2018), 1807.09769.

[45] P. Fileviez Pérez, C. Murgui, and A. D. Plascencia, JHEP 11, 093 (2019), 1908.01772.

[46] P. Fileviez Pérez, C. Murgui, and A. D. Plascencia, JHEP 01, 091 (2020), 1911.05738.

[47] G. 't Hooft, NATO Sci. Ser. B 59, 135 (1980).

[48] L. L. Everett and O. Popov, (2021), 2108.09316.

[49] S. Weinberg, Phys. Lett. B 91, 51 (1980).

[50] L. J. Hall, Nucl. Phys. B 178, 75 (1981). 